have sex with men. For populations with 3 or fewer prevalence data points Spectrum-STI uses time constant prevalence ratios from the WHO 2016 global STI estimates. Population size estimates were from Brazil's National Survey of Knowledge, Attitudes and Practices of HIV and other STIs (PCAP-2013).

Results We identified 48 studies that met the study entry criteria providing 47 data points for women and 7 for men. After excluding studies done exclusively in HIV positive individuals or individuals seeking STI treatment we had 29 data points for women and 6 for men. The Spectrum-STI national prevalence estimate for 2020 for adult women was $0.63 \%(0.13-$ 2.23) and for men $0.70 \%(0.16-2.44)$.

Conclusion The prevalence of urogenital gonorrhoea in Brazil appears to be low. Data on the prevalence in men, however, are very limited. The results from this study are being used to inform the design and prioritization of new surveillance surveys.

\section{P382 HIGH PREVALENCE OF MACROLIDE AND QUINOLONE- RESISTANCE MEDIATING MUTATIONS IN MYCOPLASMA GENITALIUM AMONG GAY AND BISEXUAL MEN (GBM) IN MONTRÉAL, CANADA}

\begin{abstract}
${ }^{1,2} \mathrm{~A}$ Labbé ${ }^{*},{ }^{3,4} \mathrm{G}$ Lambert, ${ }^{1,5} \mathrm{C}$ Fortin, ${ }^{3} \mathrm{~A}$ Fourmigue, ${ }^{3} \mathrm{H}$ Apelian, ${ }^{3} \mathrm{M}$ Dvorakova, ${ }^{6} \mathrm{D}$ Moore, ${ }^{7} \mathrm{~N}$ Lachowsky, ${ }^{8} \mathrm{~J}$ Jollimore, ${ }^{9} \mathrm{D}$ Grace, ${ }^{9,10} \mathrm{~T}$ Hart, ${ }^{11}$ I Martin, ${ }^{3,12} \mathrm{~J}$ Cox, Engage Study Group. 'Université de Montréal, Montréal," Canada; ${ }^{2}$ Division of Infectious Diseases and Microbiology Hôpital Maisonneuve-Rosemont, Montréal, Canada; ${ }^{3}$ Direction Régionale de Santé Publique de Montréal, Montréal, Canada; ${ }^{4}$ nstitut National de Santé Publique du Québec, Montréal, Canada; ${ }^{5}$ Division of Infectious Diseases and Microbiology, Centre Hospitalier de I'Université de Montréal, Montréal, Canada; ${ }^{6} B C$ Centre for Excellence in HIVIAIDS, Vancouver," Canada; 'University of Victoria, Victoria," Canada; ${ }^{8}$ CommunityBased Research Centre, Vancouver, Canada; ${ }^{9}$ University of Toronto, Toronto," Canada; ${ }^{10}$ Ryerson University, Toronto," Canada; ${ }^{11}$ National Microbiology Laboratory, Public Health Agency of Canada, Winnipeg, Canada; ${ }^{12}$ Mc Gill University, Montréal, Canada
\end{abstract}

10.1136/sextrans-2021-sti.417

Background Mycoplasma genitalium (MG) easily develops resistance to azithromycin and moxifloxacin, currently recommended first- and second-line treatments, respectively. Population-based data on prevalence of MG resistance mutations (macrolide and fluoroquinolone) are lacking. We estimated this prevalence among GBM.

Methods The Engage study used respondent-driven sampling (RDS), to recruit sexually active cisgender and transgender men, $\geq 16$ years. Participants completed a computer-assisted self-interview. Pharyngeal, urine and rectal specimens collected at cohort study visits between 11/2018-11/2019 were analyzed using Seegene Allplex ${ }^{\mathrm{TM}}$ CT/NG/MG/TV assay. MG positive samples were further analyzed using Seegene Allplex ${ }^{\mathrm{TM}}$ MG \& AziR and Allplex ${ }^{\mathrm{TM}}$ MG \& MoxiR assays.

Results MG infection was detected in 44/717 participants. Resistance assays were performed on samples from 41 participants; median age $=31 \mathrm{yrs}, 78 \%$ identified as gay, $17 \%$ were HIV-positive, and 20\% reported C. trachomatis or N. gonorrhea infection over the past 6 months. Information on symptoms at study visit was available for 33 participants; all were asymptomatic. Sites of infection were rectum $(n=23)$, urethra $(n=16)$ or pharynx $(n=2)$. Macrolide-resistance mediating mutations (MRMM) in 23S rRNA gene were found in 31 samples (A2058G, $n=4$; A2059G, $n=27$ ); 6 were wild-type and 4 failed to amplify MG. Prevalence of MRMM was 31/ 37 (84\%). Quinolone-resistance mediating mutations (QRMM) in parC gene were found in (G248T, $n=10 ;$ G248A, $n=1$; A247C, $n=1) ; 25$ were wild-type and 4 failed to amplify MG. No QRMM in gyrA gene was found. Prevalence of QRMM was 12/37 (32\%). Combined mutations in 23S rRNA and parC genes was found in 11/35 MG-positive samples (31\%).

Conclusions Among asymptomatic MG-infected GBM in Montreal, almost one-third were infected by MG strains harboring resistance mutations to both antibiotics currently used to treat symptomatic infections. It is important that clinicians be aware of this high level of circulating resistance, have increased access to MG testing, and adjust their treatment strategies accordingly.

\section{P383 IS SYNDROMIC MANAGEMENT APPROACH OF STI ADEQUATE AMONG HIV POSITIVE KEY POPULATION? SYSTEMATIC DATA REVIEW IN NAIROBI KENYA}

${ }^{1} \mathrm{M}$ Akolo*, 1,2J Kimani, 1,2 L Gelmon. 'PHDP, Nairobi, Kenya; ${ }^{2}$ University of Manitoba, Nairobi, Kenya

\subsection{6/sextrans-2021-sti.418}

Background Sex workers Outreach Program (SWOP) is an STI/HIV research center serving 35000 female sex workers (FSW) through outreach services with 18000 seek HIV/STI services from the seven SWOP facilities spread across Nairobi Kenya. Program analysis showed high use of STI syndromic management antibiotics compared to budgetary allocations. This study was done to identify the gap

Methodology Systematic data review of HIV positive FSW attending SWOP clinics and having been treated STI through syndromic management was done for the last two years 2020 and 2019. Data was pulled for all female sex workers who had been managed for STI syndromically, HIV status was checked and those who had a HIV diagnosis were retained in the study data base (this is because the HIV positive frequent the sites at least four times in a year compared to HIV negative FSW) Data was then separated into one time STI treatment and revisits with the same signs and symptoms for more than once in one year. Data on consistence condom use was also extracted

Results Out of the 17,900 Female sex workers accessing SWOP facilities for key population services, 3038(16.9) were HIV positive. Of this HIV positive at least 4\% (122) were treated for STI through syndromic management with highest $66 \%$ (81) reporting low abdominal pains with vaginal discharge. $52 \%$ (64) come back with same symptom twice of which 79\%(51) reported consistent condom use, while 23\% (15) come back at least more than twice with the same complains within a year with $89 \%$ reporting consistent condom use.

Conclusion Given the nature of work, female sex workers have a high possibility of STI misdiagnosis. Hence countries should strive to invest in laboratory screening of STI among female sex workers to improve on correct diagnosis and treatment to reduce unnecessary use of antibiotics 